\title{
EVALUACIÓN DE LOS TRASTORNOS ALIMENTARIOS: PROPIEDADES PSICOMÉTRICAS DEL TEST EDI-2 EN ADOLESCENTES ESCOLARIZADOS(AS) DE 13 A 18 AÑOS
}

\author{
EVALUATION OF EATING DISORDERS: \\ PSYCHOMETRIC PROPERTIES OF EDI-2 IN \\ STUDENTS 13 TO 18 YEARS OLD
}

\author{
Alfonso Urzúa M., Sandy Castro R., America Lillo O., Carolina Leal P.
}

Escuela de Psicología, Universidad Católica del Norte, Antofagasta, Chile

\begin{abstract}
Objective: To analyze the psychometric properties of the test EDI-2 to evaluate eating disorders in Chilean adolescents Method: The sample was constituted by 1,429 adolescents (595 men and 835 women) between 13 and 18 years from the city of Antofagasta, to whom the EDI-2 test was applied. The reliability and the validity of the instrument were evaluated by factorial analysis and t-test. Results: The majority of the dimensions and the total scale have alphas above 0.70. Factorial structure observed was similar to the theoretical proposal. Conclusions: The EDI-2 test with corrections applied to Chilean population is a reliable and valid instrument for use as a screening tool in early detection of eating disorders.
\end{abstract}

Key words: EDI-2, eating disorders, validity, adolescents.

Este trabajo fue recibido el 10 de Marzo de 2009 y aceptado para ser publicado el 20 de Junio de 2009.

\section{INTRODUCCIÓN}

Los trastornos de conducta alimentaria (TCA) son desórdenes complejos que comprenden dos tipos de alteraciones conductuales: unos directamente relacionados con la comida y el peso y otros derivados de la relación consigo mismo y con los demás. Estos trastornos se han constituido en una patología emergente en los países desarrollados y en vías de desarrollo, configurándose como la tercera enfermedad crónica más común entre los adolescentes después de la obesidad y el asma (1).

Los TCA se presentan cada vez en edades más tempranas, descendiendo en 12 meses la edad de aparición en la última década y manteniendo a la vez su prevalencia en edades más avanzadas. Esto ha implicado que el rango etáreo aumente, haciendo que los TCA sean etiquetados como epidémicos en sociedades industrializadas.

Este aumento en la demanda ha generado una necesidad creciente de la atención de profesionales de la salud, los cuales se encuentran atendiendo adolescentes con mucha más frecuencia y que, sumados a la posibilidad diagnóstica de un trastorno alimentario concomitante con diversos factores tales como: sociales, afectivos, comorbilidad con otros trastornos, entre otros han generado una respuesta no del todo exitosa en la salud pública.

La posibilidad de contar con un diagnóstico certero que considere los diversos signos y conductas que presentan las personas que padecen este tipo de trastornos, posibilitaría por un lado la optimización del recurso humano y fundamentalmente, pesquisar precozmente a aquellas personas que se encuentren en un punto de riesgo, facilitando la labor preventiva. Se suma a esto que un inicio precoz del tratamiento optimiza su respuesta y favorece un mejor pronóstico (2).

Es en este contexto que en los últimos años se han creado numerosos instrumentos para la exploración de los mismos. Actualmente existen diversas herramientas al servicio de los distintos profesionales que han sido construidos para la detección de estos trastornos como por ejemplo: Eating Attitudes Test (EAT), Bulimia Test Revised (BULIT-R), Bulimia Test - Revised (BULIT-R), Questionnaire of Eating and Weight Patterns-Revised 
(QEWP-R), BSQ, Eating Disorder Examination-selfreport questionnaire (EDE-Q), Eating Disorder Inventory (EDI), EDI- 2, entre otros.

Todos estos cuestionarios que facilitan un diagnóstico o una exploración frente a los TCA, han sido construidos y validados en países con culturas y personalidades muy distintas a la de nuestra sociedad y principalmente en países de habla inglesa. Usualmente estos cuestionarios son traducidos a otros idiomas sin cuidar los procedimientos adecuados y sin evaluar posteriormente su confiabilidad y validez, constituyéndose así el lenguaje en un factor entorpecedor al momento de la aplicación e interpretación de estos, ya que la semántica varía de cultura en cultura.

La presente investigación se enfocó a analizar las propiedades psicométricas del Inventario de Trastornos de la Conducta Alimentaria en su segunda versión EDI-2- (del inglés Eating Disorders Inventory), un cuestionario cuya versión en castellano fue adaptada y publicada en España (3). Esta versión en castellano fue validada en España para adolescentes entre 13 y 18 años y cuenta con una serie de publicaciones que avalan su validez en el campo de los trastornos alimentarios $(4,5)$.

Este instrumento ya se ha comenzado a utilizar en Chile en estudios de prevalencia $(6,7)$ y en otras investigaciones sobre trastornos alimentarios (8), sin embargo, no han reportado su comportamiento psicométrico en tanto confiabilidad y validez en adolescentes chilenos.

La relevancia de esta investigación recae en que su utilidad metodológica e implicancia práctica aportará no solo a profesionales psicólogos sino que también al área de la salud pública y educacional, ya que será una herramienta adecuada y de fácil acceso para poder pesquisar con mayor precisión este tipo de trastornos en nuestra sociedad.

Esta investigación adquiere además relevancia social por el hecho de relevar si el instrumento se constituye o no como una buena metodología para realizar estudios de prevalencia en el país.

El objetivo de esta investigación fue analizar las propiedades psicométricas del EDI-2 en adolescentes chilenos. Se espera encontrar diferencias significativas entre hombres y mujeres, lo cual aportará evidencias sobre la capacidad del instrumento para discriminar entre grupos que la investigación en el tema ha reportado como diferentes (3).

\section{SUJETOS Y MÉTODO Participantes}

Se consideró una muestra no clínica de tipo intencional estratificada que comprendió a 595 hombres (42\%) y 835 mujeres $(58 \%)(n=1430)$, los cuales cursaban entre primero y cuarto medio tanto de colegios municipales, subvencionados como privados de la ciudad de Antofagasta.

\section{Instrumento}

El Eating Disorder Inventory (EDI) fue creado por David Garner en 1983. Esta es una escala de auto-reporte que mide características psicológicas y sintomatología asociada a anorexia y bulimia nerviosa. Inicialmente contaba con 64 ítems en 8 subescalas a las cuales 10 años después se le adicionaron 27 ítems en 3 subescalas configurándose como el EDI-2 (9).

La actual versión (EDI-2) en su traducción al español consiste en 91 reactivos con un formato de respuestas en una escala tipo Likert de seis puntos: "siempre", "casi siempre", "frecuentemente", "en ocasiones", "rara vez" o "nunca" que permiten puntuar en 11 escalas, 8 principales (obsesión por las delgadez (DT); bulimia (B); insatisfacción corporal (BD); ineficacia (I); perfeccionismo (P); desconfianza interpersonal (ID); conciencia introceptiva (IA) y miedo a la madurez (MF)) y 3 adicionales (ascetismo (A), impulsividad (IR) e inseguridad social (SI)) (10).

En cuanto a la consistencia interna del instrumento, la fiabilidad evaluada a través del Alfa de Cronbach en el estudio de Garner (11) oscila entre 0.83 y 0.93 en las distintas categorías, arrojando valores similares en la muestra clínica en la validación de Corral et al (2006), con valores levemente inferiores en la muestra normal.

La utilización de este instrumento ha demostrando ser un buen indicador para discriminar entre pacientes con trastorno alimentario y grupo control normal como se observa en la validación realizada en Suecia en donde se encontró que mujeres con TCA, pacientes y no pacientes, puntuaban significativamente alto en síntomas y características personales en comparación con mujeres $\sin$ TCA (12).

\section{Procedimientos}

A fin de resguardar los aspectos éticos, la investigación fue aprobada por los Comités de Investigación tanto del área clínica de la Escuela de Psicología, como por el de la Corporación Municipal de Desarrollo Social, quien posteriormente autorizó el ingreso a los establecimientos municipales. Se realizó en primera instancia una aplicación piloto a 50 sujetos, 19 hombres y 31 mujeres, con una media de edad de 12.44 años (D.E.=1.26), con el fin de adaptar el lenguaje de las preguntas al contexto cultural. En esta fase piloto se evaluó la equivalencia de contenido y semántica. Una vez aplicado el instrumento, 
se les solicitó a los adolescentes mediante entrevistas grupales retrospectivas, que evaluaran el grado de comprensión y aceptabilidad de la traducción del castellano español. Se recogieron las preguntas, dudas y comentarios de los adolescentes sobre el grado de dificultad de las preguntas y su comprensión, inquiriendo, por ejemplo, si hubo frases o palabras difíciles de entender, difíciles de responder o que no eran pertinentes. Con dicha información, se modificó la redacción y el vocabulario del cuestionario para mejorar la comprensión de la prueba, agregándose entre paréntesis sinónimos o ejemplos en seis de los reactivos originales (anexo 1). Todas las preguntas fueron modificadas con su versión en femenino (Ej.: me siento solo, quedo redactado me siento solo (a) ).

Para realizar la recolección de los datos se solicitó además la autorización a la Dirección de cada establecimiento educacional. La aplicación del cuestionario final se realizó de forma masiva en cada sala de clases durante la jornada escolar, de acuerdo a la disponibilidad de cada curso. Se solicitó previamente el asentimiento a todos los adolescentes que participaron en el estudio. El tiempo de respuesta tomó de 25 a 45 minutos.

Una vez recogidos los datos estos fueron ingresados en la base de datos SPSS 11.5 para realizar los análisis estadísticos respectivos.

\section{Análisis estadístico}

Para evaluar la fiabilidad se consideró el análisis de la consistencia interna mediante el estadístico Alfa de Cronbach. La validez de constructo se analizó mediante la realización de un análisis factorial de los componentes principales con rotación oblicua (OBLIMIN), dada la relación teórica existente entre las variables. El análisis factorial confirmatorio se realizó con el programa LISREL 8.30. Como medida de validez discriminante se realizaron análisis de diferencias de medias por sexo a través de la prueba t de student.

\section{RESULTADOS \\ Participantes}

Participaron 1430 sujetos escolarizados entre 13 y 18 años de edad con una media de 15.55 años (D.E.= 1.21). La distribución de los sujetos por curso fue de un $38 \%$ de primero medio, $22 \%$ segundo medio, $27.6 \%$ de tercero medio y un $12.3 \%$ en cuarto medio.

El $42 \%$ asistía a colegios municipales, un $73 \%$ a establecimientos subvencionados y un $21 \%$ a establecimientos privados. La distribución por sexo, tipo de establecimiento y rango de edad se presenta en la tabla 1.

\section{Fiabilidad}

Al calcular la consistencia interna mediante el estadístico alfa de Cronbach, se encuentra que con excepción de las dimensiones miedo a la madurez, bulimia y perfeccionismo, todas las escalas presentan un alfa sobre 0.70 , superando incluso el 0.80 a nivel de la escala total (anexo 2).

\section{Análisis de ítems}

Al analizar la correlación ítem total menos el ítem, se encuentra un valor máximo alcanzado de $0.50 ; 42$ de

\section{ANEXO 1}

\section{Modificaciones realizadas al EDI-2}

\section{Reactivos originales EDI-2}

1-. Como dulces e hidratos de carbono sin preocuparme

5.- Suelo hartarme de comida

38.- Suelo pensar en darme un atracón

70-. Digo impulsivamente cosas de las que después me arrepiento

86-. Me siento incómodo por las necesidades de mi cuerpo

\section{Reactivos modificados estudio Chile}

1-. Como dulces e hidratos de carbono (pan, fideos) sin preocuparme

5.- Suelo hartarme (llenarme) de comida

38.- Suelo pensar en darme un atracón (cantidad inusualmente grande de comida)

70-. Digo impulsivamente ( $\sin$ pensar) cosas de las que después me arrepiento

86-. Me siento incómodo (a) por las necesidades de mi cuerpo (orinar, defecar) 
los reactivos presentan una correlación menor a 0.30 . Estos índices de correlación mejoran sustantivamente, especialmente en aquellos con valores inferiores a $0.1 \mathrm{o}$ los negativos, al calcular la correlación ítem dimensión (anexo 2).

\section{Indicaciones de validez Constructo}

Tal como se puede observar en la tabla 2, todas las dimensiones del EDI-2 se encuentran interrelacio- nadas.

Dada la relación teórica existente entre las variables, se opta por realizar un análisis factorial a través del método de componentes principales con rotación OBLIMIN. La medida de adecuación muestral de Kaiser - Meyer - Olkin fue de 0.90, permitiendo la realización de un análisis factorial. La prueba de esfericidad de Bartlett permite rechazar la hipótesis de matrices similares, existiendo por tanto correlación entre los datos $\left(\chi^{2}(4095\right.$, $\mathrm{N}=1429)=27173, \mathrm{p}<0.001)$

\section{TABLA 1}

Distribución de la muestra por sexo, rango de edad y por tipo de establecimiento.

\begin{tabular}{lcccccc} 
& \multicolumn{2}{c}{$\mathbf{1 3}$ a $\mathbf{1 4}$ años } & \multicolumn{2}{c}{$\mathbf{1 5}$ a 16 años } & \multicolumn{2}{c}{$\mathbf{1 7}$ a $\mathbf{1 8}$ años } \\
& H $(\boldsymbol{\%})$ & $\mathbf{M}(\boldsymbol{\%})$ & H $(\boldsymbol{\%})$ & $\mathbf{M}(\boldsymbol{\%})$ & H $(\boldsymbol{\%})$ & M $(\boldsymbol{\%})$ \\
\hline Público & $58(16.1)$ & $123(34.1)$ & $103(14.5)$ & $162(22.8)$ & $66(18.5)$ & $88(24.6)$ \\
Subvencionado & $40(11.1)$ & $72(19.9)$ & $118(16.6)$ & $163(22.8)$ & $66(18.5)$ & $72(20.2)$ \\
Privado & $26(7.2)$ & $42(11.6)$ & $82(11.5)$ & $84(11.8)$ & $36(10.1)$ & $29(8.1)$ \\
Total & $124(34.3)$ & $237(65.7)$ & $303(42.6)$ & $409(57.4)$ & $168(47.1)$ & $189(52.9)$ \\
Total grupo edad & \multicolumn{2}{c}{$361(100)$} & \multicolumn{2}{c}{$712(100)$} & $357(100)$
\end{tabular}

$\mathrm{H}=$ hombres; $\mathrm{M}=$ mujeres; $(\%)=$ porcentaje observado con relación al total grupo edad

\begin{tabular}{|c|c|c|c|}
\hline \multicolumn{4}{|c|}{ ANEXO 2} \\
\hline \multicolumn{4}{|c|}{ Correlaciones item total - Ítem dimensión - $\alpha$ de Cronbach por dimensión } \\
\hline Factor $/ \mathbf{N}^{0}$ reactivo & $\begin{array}{c}\text { Correlación ítem } \\
\text { Total }\end{array}$ & $\begin{array}{l}\text { Correlación ítem } \\
\text { Dimensión }\end{array}$ & $\begin{array}{l}\alpha \text { de Cronbach } \\
\text { dimensión }\end{array}$ \\
\hline \multicolumn{4}{|c|}{ Obsesión por la delgadez } \\
\hline 1 & 0.02 & 0.13 & \\
\hline 7 & 0.48 & 0.65 & \\
\hline 11 & 0.48 & 0.62 & \\
\hline 16 & 0.43 & 0.63 & 0.83 \\
\hline 25 & 0.48 & 0.64 & \\
\hline 32 & 0.56 & 0.67 & \\
\hline 49 & 0.47 & 0.68 & \\
\hline \multicolumn{4}{|l|}{ Bulimia } \\
\hline 4 & 0.31 & 0.38 & \\
\hline 5 & 0.20 & 0.39 & \\
\hline 28 & 0.21 & 0.39 & \\
\hline 38 & 0.19 & 0.46 & 066 \\
\hline 46 & 0.26 & 0.39 & \\
\hline 53 & 0.44 & 0.29 & \\
\hline 61 & 0.25 & 0.31 & \\
\hline \multicolumn{4}{|l|}{ Insatisfacción corporal } \\
\hline 2 & 0.34 & 0.33 & \\
\hline 9 & 0.41 & 0.49 & \\
\hline 12 & 0.41 & 0.49 & \\
\hline
\end{tabular}




\begin{tabular}{|c|c|c|c|}
\hline Factor $/ \mathbf{N}^{0}$ reactivo & $\begin{array}{c}\text { Correlación ítem } \\
\text { Total }\end{array}$ & $\begin{array}{c}\text { Correlación ítem } \\
\text { Dimensión }\end{array}$ & $\begin{array}{c}\alpha \text { de Cronbach } \\
\text { dimensión }\end{array}$ \\
\hline 19 & 0.48 & 0.54 & \\
\hline 31 & 0.25 & 0.31 & 0.77 \\
\hline 45 & 0.42 & 0.49 & \\
\hline 55 & 0.39 & 0.55 & \\
\hline 59 & 0.30 & 0.34 & \\
\hline 62 & 0.38 & 0.57 & \\
\hline \multicolumn{4}{|l|}{ Miedo a la madurez } \\
\hline 3 & 0.37 & 0.36 & \\
\hline 6 & 0.31 & 0.29 & \\
\hline 14 & 0.01 & 0.32 & \\
\hline 22 & -0.04 & 0.28 & 0.62 \\
\hline 35 & 0.27 & 0.29 & \\
\hline 39 & 0.70 & 0.33 & \\
\hline 48 & 0.31 & 0.40 & \\
\hline 58 & 0.09 & 0.28 & \\
\hline \multicolumn{4}{|l|}{ Conciencia introceptiva } \\
\hline 8 & 0.44 & 0.47 & \\
\hline 21 & 0.31 & 0.42 & \\
\hline 26 & 0.17 & 0.12 & \\
\hline 33 & 0.33 & 0.36 & \\
\hline 40 & 0.26 & 0.25 & \\
\hline 44 & 0.42 & 0.51 & 0.71 \\
\hline 47 & 0.32 & 0.27 & \\
\hline 51 & 0.37 & 0.46 & \\
\hline 60 & 0.37 & 0.50 & \\
\hline 64 & 0.46 & 0.39 & \\
\hline \multicolumn{4}{|l|}{ Ineficacia } \\
\hline 10 & 0.34 & 0.32 & \\
\hline 18 & 0.33 & 0.43 & \\
\hline 20 & 0.26 & 0.34 & \\
\hline 24 & 0.43 & 0.46 & \\
\hline 27 & 0.42 & 0.52 & \\
\hline 37 & 0.42 & 0.53 & \\
\hline 41 & 0.44 & 0.54 & \\
\hline 42 & 0.28 & 0.43 & \\
\hline 50 & 0.28 & 0.48 & \\
\hline 56 & 0.37 & 0.41 & \\
\hline \multicolumn{4}{|l|}{ Perfeccionismo } \\
\hline 13 & 0.19 & 0.36 & \\
\hline 29 & 0.19 & 0.29 & \\
\hline 36 & 0.33 & 0.31 & 0.58 \\
\hline 43 & 0.15 & 0.35 & \\
\hline 52 & 0.20 & 0.36 & \\
\hline 63 & 0.13 & 0.26 & \\
\hline \multicolumn{4}{|c|}{ Desconfianza interpersonal } \\
\hline 15 & 0.22 & 0.51 & \\
\hline 17 & 0.13 & 0.32 & \\
\hline 23 & 0.20 & 0.38 & 0.66 \\
\hline 30) & 0.12 & 0.35 & \\
\hline 34 & 0.29 & 0.31 & \\
\hline 54 & 0.24 & 0.25 & \\
\hline 57 & 0.20 & 0.42 & \\
\hline
\end{tabular}


El análisis aporta una solución de 8 factores que explican un porcentaje bajo de la varianza (38.45\%). La distribución de las cargas factoriales por dimensión pueden ser observados en la tabla 3.

Se observó que 10 de los reactivos puntearon más alto en factores distintos a los que corresponderían según la propuesta española.

La tabla 4 muestra los índices de ajuste del análisis factorial confirmatorio para el modelo de 8 factores y el modelo de 11 factores (adicionando las tres escalas adicionales). Tal como se observa, el índice de ajuste de chi cuadrado indica el no ajuste de los datos a la estructura factorial estudiada, pero tal como señala Russell (13) el test de chi cuadrado como medida de bondad de ajuste está muy influido por el tamaño de muestra, por lo que también se han proporcionado otros índices de ajuste.

Los índices CFI (Comparative Fit Index), RFI (Relative Fit Index) son inferiores a 0.90 en ambos modelos, valor considerado como buen ajuste o superior (14). Pese a esto, estos indicadores se acercan más a dicho valor en el modelo de 8 factores.

Algo similar ocurre con el índice de bondad de ajuste GFI, que representa el grado de ajuste conjunto (valores entre $0=$ mal ajuste a $1=$ ajuste perfecto), en donde el valor más cercano a 1 es aquel encontrado al evaluar el modelo de ocho factores.

El índice RMSEA (Root Mean Square Error of Approximation), que resulta significativo con valores

\begin{tabular}{|c|c|c|c|c|c|c|c|c|}
\hline \multicolumn{9}{|c|}{ TABLA 2} \\
\hline \multicolumn{9}{|c|}{ Correlaciones bivariadas dimensiones del EDI-2 } \\
\hline & & 2 & 3 & 4 & 5 & 6 & 7 & 8 \\
\hline \multicolumn{9}{|l|}{ 1. Obsesión } \\
\hline \multirow[t]{3}{*}{ por la delgadez } & $\mathrm{r}$ & $0.191(* *)$ & $0.544(* *)$ & $0.135(* *)$ & $0.363(* *)$ & ,312(**) &, $194(* *)$ &, $076(* *)$ \\
\hline & $\mathrm{p}$ & 0.000 & 0.000 & 0.000 & 0.000 & 0.000 & 0.000 & 0.000 \\
\hline & $\mathrm{N}$ & 1370 & 1347 & 1357 & 1358 & 1343 & 1344 & 1369 \\
\hline \multirow[t]{3}{*}{ 2. Bulimia } & $\mathrm{r}$ & & $0.234(* *)$ & $0.146(* *)$ & $0.454(* *)$ & $0.241(* *)$ & $0.259(* *)$ & 0.089 (**) \\
\hline & $\mathrm{p}$ & & 0.000 & 0.000 & 0.000 & 0.000 & 0.000 & 0.001 \\
\hline & $\mathrm{N}$ & & 1341 & 1355 & 1353 & 1340 & 1343 & 1366 \\
\hline \\
\hline \multirow[t]{3}{*}{ corporal } & $\mathrm{r}$ & & & $0.131(* *)$ & $0.363(* *)$ & 0.449 (**) & $0.117(* *)$ & $0.224(* *)$ \\
\hline & $\mathrm{p}$ & & & 0.000 & 0.000 & 0.000 & 0.000 & 0.000 \\
\hline & $\mathrm{N}$ & & & 1328 & 1333 & 1311 & 1318 & 1340 \\
\hline \multicolumn{9}{|l|}{ 4. Miedo a } \\
\hline \multirow[t]{3}{*}{ la madurez } & $\mathrm{r}$ & & & & $0.225(* *)$ & $0.136(* *)$ & $0.157(* *)$ & 0.040 \\
\hline & $\mathrm{p}$ & & & & 0.000 & 0.000 & 0.000 & 0.142 \\
\hline & $\mathrm{N}$ & & & & 1346 & 1334 & 1334 & 1351 \\
\hline \multicolumn{9}{|l|}{ 5. Conciencia } \\
\hline \multirow[t]{3}{*}{ introceptiva } & $\mathrm{r}$ & & & & & $0.461(* *)$ & $0.266(* *)$ & $0.268(* *)$ \\
\hline & $\mathrm{p}$ & & & & & 0.000 & 0.000 & 0.000 \\
\hline & $\mathrm{N}$ & & & & & 1328 & 1332 & 1353 \\
\hline \multirow[t]{3}{*}{ 6. Ineficacia } & $\mathrm{r}$ & & & & & & $0.145(* *)$ & $0.414(* *)$ \\
\hline & $\mathrm{p}$ & & & & & & 0.000 & 0.000 \\
\hline & $\mathrm{N}$ & & & & & & 1318 & 1335 \\
\hline \multirow[t]{3}{*}{ 7. Perfeccionismo } & $\mathrm{r}$ & & & & & & & 0.006 \\
\hline & $\mathrm{p}$ & & & & & & & 0.834 \\
\hline & $\mathrm{N}$ & & & & & & & 1340 \\
\hline \multirow{4}{*}{$\begin{array}{l}\text { 8. Desconfianza } \\
\text { interpersonal }\end{array}$} & & & & & & & & \\
\hline & $\mathrm{r}$ & & & & & & & 1 \\
\hline & $\mathrm{p}$ & & & & & & & . \\
\hline & $\mathrm{N}$ & & & & & & & 1392 \\
\hline
\end{tabular}


TABLA 3

\section{Estructura factorial}

\begin{tabular}{|c|c|}
\hline Reactivos & 1 \\
\hline \multicolumn{2}{|l|}{ Obsesión por la delgadez } \\
\hline $\begin{array}{l}\text { 1-. Como dulces e hidratos de carbono } \\
\text { (pan, fideos) sin preocuparme }\end{array}$ & $0.191^{*}$ \\
\hline 7.- Pienso en ponerme a dieta & 0.758 \\
\hline 11.- Me siento muy culpable cuando como en exceso & 0.714 \\
\hline 16.- Me aterroriza la idea de engordar & 0.743 \\
\hline $\begin{array}{l}\text { 25.- Exagero o doy demasiada } \\
\text { importancia al peso }\end{array}$ & 0.749 \\
\hline $\begin{array}{l}\text { 32.- Estoy preocupado (a) porque } \\
\text { querría ser una persona más delgada }\end{array}$ & 0.760 \\
\hline $\begin{array}{l}\text { 49.- Si engordo un kilo, me preocupa } \\
\text { que pueda seguir ganando peso }\end{array}$ & 0.787 \\
\hline
\end{tabular}

\section{Bulimia}

4.- $\quad$ Suelo comer cuando estoy disgustado (a)

5.- $\quad$ Suelo hartarme (llenarme) de comida

28.- He ido a comilonas en las que sentí que no podía parar de comer

$2-3$

3

4

5

6

7

38.- Suelo pensar en darme un atracón (cantidad inusualmente grande de comida)

46.- Como con moderación delante de los demás, pero me doy un atracón cuando se van.

53.- Pienso en vomitar para perder peso

61.- Como o bebo a escondidas

Insatisfacción corporal

2-. Creo que mi estómago es demasiado grande

0.385

9.- Pienso que mis muslos son demasiado gruesos

12.- Creo que mi estómago tiene el tamaño adecuado

19.- Me siento satisfecho (a) con mi figura

31.- Me gusta la forma de mi trasero

45.- Creo que mis caderas son demasiado anchas

55.- Creo que el tamaño de mis muslos es adecuado

59.- Creo que mi trasero es demasiado grande

62.- Creo que mis caderas tienen el tamaño adecuado

\section{Miedo a la madurez}

3.- Me gustaría volver a ser niño (a) para sentirme seguro (a)

6.- Me gustaría ser más joven

14.- La infancia es la época más feliz de mi vida

22.- Preferiría ser adulto (a) a ser niño (a)

35.- Las exigencias de la vida adulta son excesivas

48.- Creo que las personas son más felices cuando son niños

58.- Los mejores años de tu vida son cuando llegas a ser adulto

\section{Conciencia introceptiva}

8.- Me asusto cuando mis sentimientos son muy fuertes 


\section{Estructura factorial}

\section{Reactivos}

1

2

3

4

5

6

7

8

21.- Suelo estar confuso (a)

sobre mis emociones

0.596

26-. Puedo reconocer las emociones

que siento en cada momento

0.452

$0.160 *$

33.- No sé qué es lo que ocurre en mi interior

0.481

40-. No sé muy bien cuando tengo hambre o no

$0.412 \quad 0.220 *$

0.670

44.- Temo no poder controlar mis sentimientos

$0.242 *$

de una comida normal

triste, asustado (a) o enfadado (a)

60.- Tengo sentimientos que no puedo identificar del todo

64-. Cuando estoy disgustado (a) temo empezar a comer

\section{Ineficacia}

10.- Me considero una persona poco eficaz

18.- Me siento solo (a) en el mundo

20-. Creo que generalmente controlo las cosas que me pasan en la vida

24.- Me gustaría ser otra persona

27.- Me siento incapaz

37-. Me siento seguro (a) de mi mismo (a)

41.- Tengo mala opinión de mí

42.- Creo que puedo conseguir mis objetivos

50.- Me considero una persona valiosa

56.- Me siento emocionalmente vacío (a) en mi interior

\section{Perfeccionismo}

13.- En mi familia sólo se consideran suficientemente buenos los resultados sobresalientes

29.- Cuando era pequeño (a), intentaba con empeño no decepcionar a mis padres y profesores

36.- Me fastidia no ser el (la) mejor en todo

43.- Mis padres esperaban de mí resultados sobresalientes

52.- Creo que debo hacer las cosas perfectamente o no hacerlas

63.- Me fijo objetivos sumamente ambiciosos

\section{Desconfianza interpersonal}

15.- Soy capaz de expresar mis sentimientos

17.- Confío en los demás

23.- Me resulta fácil comunicarme con los demás

30.- Tengo amigos (as) íntimos (as)

34-. Me cuesta expresar mis emociones a los demás

54.- Necesito mantener cierta distancia con la gente; me siento incómodo si alguien se acerca demasiado $\quad 0.305$

57.- Soy capaz de hablar sobre aspectos personales y sentimientos 
inferiores a 0.08 (15) alcanza este valor en ambos modelos factoriales.

A partir estos índices de ajuste se puede decir que el ajuste de los datos a la estructura factorial es moderada, y ligeramente superior en el modelo de 8 factores.

\section{Capacidad de discriminación}

\section{Sexo}

En cuanto a la validez discriminante se utilizó el procedimiento de pruebas $\mathrm{T}$ para muestras independientes para evaluar la diferencia entre las medias encontradas para ambos sexos (tabla 5).
Se observaron diferencias estadísticamente significativas en las dimensiones obsesión por la delgadez $\left(\mathrm{t}_{(\mathrm{g} . \mathrm{l}=1399)}=-12,286 ; \mathrm{p}<0.001\right)$, insatisfacción personal $\left(\mathrm{t}_{\mathrm{g} . \mathrm{l}=1364)}=-6,818 ; \mathrm{p}<0.001\right)$, conciencia introceptiva $\left(\mathrm{t}_{(\mathrm{g}}\right.$ $\mathrm{l}=1380)=-4,430 ; \mathrm{p}<0.001)$ e ineficacia $\left(\mathrm{t}_{(\mathrm{g} . \mathrm{l}) 1363)}=-3,604\right.$; $\mathrm{p}<0.001)$.

\section{DISCUSIÓN}

$\mathrm{Al}$ evaluar la consistencia interna del EDI-2, el $\alpha$ encontrado para la prueba total permitiría su uso como instrumento de tamizaje e incluso en contextos clínicos como medida de cambio individual (16). Dado el valor

\section{TABLA 4}

Índices de ajuste para modelos factorial de ocho y once dimensiones

\begin{tabular}{lcccccc} 
Escala & $\boldsymbol{\chi}^{\mathbf{2}}$ & CFI & NFI & GFI & RFI & RMSEA \\
\hline 8 dimensiones & 7125.155 & 0.738 & 0.675 & 0.838 & 0.659 & 0.0501 \\
11 dimensiones & 12890.267 & 0.699 & 0.618 & 0.803 & 0.604 & 0.0461
\end{tabular}

$\chi^{2}=$ Normal Theory Weighted Least Squares Chi-Square; CFI= Comparative Fit Index; NFI= Normed Fit Index;

GFI; Goodness of Fit Index; RFI= Relative Fit Index; RMSEA= Root Mean Square Error of Approximation.

\section{TABLA 5}

Medias por dimensión según sexo

\begin{tabular}{lllll} 
& SEXO & N & Media & d.e. \\
\hline Obsesión por la delgadez & hombre & 580 & 3.9466 & 4.34667 \\
& mujer & 821 & 7.5323 & 6.00451 \\
Bulimia & hombre & 586 & 2.1126 & 2.83584 \\
& mujer & 808 & 2.1881 & 3.25371 \\
Insatisfacción corporal & hombre & 568 & 6.7183 & 5.15388 \\
Miedo a la madurez & mujer & 798 & 8.8459 & 6.03295 \\
& hombre & 578 & 10.4706 & 4.60615 \\
Conciencia introceptiva & mujer & 804 & 10.5858 & 4.30573 \\
Ineficacia & hombre & 579 & 5.1727 & 4.58649 \\
Perfeccionismo & mujer & 803 & 6.3935 & 5.36657 \\
\multirow{2}{*}{ Desconfianza interpersonal } & hombre & 569 & 4.1968 & 4.40629 \\
& mujer & 796 & 5.1281 & 4.91141 \\
& hombre & 579 & 6.6114 & 3.89471 \\
& mujer & 788 & 6.3096 & 3.87191 \\
& hombre & 579 & 5.3126 & 3.81456 \\
& mujer & 813 & 5.2251 & 3.85351
\end{tabular}


del alfa reportado por las dimensiones específicas, no se sugiere el uso de cada dimensión por separado sino como parte de la escala total.

El análisis factorial muestra que también existe una diferencia entre la escala original y este estudio ya que hay 10 ítems que puntúan de mejor manera en una dimensión distinta a la dimensión indicada inicialmente en el estudio español, aunque de todas maneras se agrupan en el factor teórico correspondiente con un menor valor. Este hecho, así como el ajuste moderado de los datos al modelo teórico tanto en la utilización de ocho factores como en el de 11, sugiere seguir profundizando el análisis de los ítems y de la estructura factorial.

Sobre la capacidad del instrumento de discriminar entre grupos teóricamente distintos, se encuentra que las diferencias en cuanto a las dimensiones como insatisfacción corporal las mujeres obtienen puntuaciones mucho más altas que los hombres, lo mismo sucede con la conciencia introceptiva. En ineficacia las mujeres puntúan también más alto que los hombres al igual que en la dimensión obsesión por la delgadez, hecho que también fue reportado por Herrera para los adolescentes españoles (4). Es posible que las mujeres le den mayor significación a temas estéticos como verse bien, y a la búsqueda incesante de la delgadez, las cuales son una característica esencial de los trastornos de la conducta alimentaria, por esto le dan mayor preocupación al peso además de contestar con mayor frecuencia en aquellos ítems que apunta al hacer dieta y un mayor miedo a engordar.

Lo anterior refleja la buena capacidad del instrumento para discriminar por sexo, al igual como ha sido demostrado en la validación española de Herrero (4).

Las limitaciones de este estudio son el no haber tenido acceso a registros reales del peso y estatura de los alumnos para haber realizado más análisis como los que aparecen en el estudio original y de traducción.

Se puede concluir entonces que las propiedades psicométricas del EDI-2 evalúan de forma adecuada y global, tendencias y comportamientos de los adolescentes entre 13 a 18 años relacionados con trastornos de la conducta alimentaria. Sin embargo, es necesario profundizar en la estructura factorial del instrumento y en la búsqueda de aquellos reactivos que aporten mayor información al constructo, dado el alto número de ítems.

\section{RESUMEN}

Objetivo: Analizar las propiedades psicométricas de la prueba EDI-2 para evaluar trastornos alimentarios en población chilena. Método: La muestra quedó constituida por 1.430 adolescentes escolarizados (595 hombres y 835 mujeres) entre 13 y 18 años, de la ciudad de Antofagasta, a los que se les aplicó la prueba EDI-2. Se analizó la fiabilidad y la validez del instrumento a través de análisis factoriales y pruebas T. Resultados: La gran mayoría de las dimensiones, así como la escala total, presentaron alfas superiores a 0,70. La estructura factorial observada fue similar a la propuesta teórica. Conclusiones: El EDI-2 aplicado con correcciones para la población Chilena es un instrumento fiable y válido para ser utilizado como herramienta de tamizaje en la detección precoz de trastornos alimentarios.

Palabras clave: EDI-2, trastorno de la conducta alimentaria, adolescentes.

Dirigir la correspondencia a:

Profesor

Alfonso Urzúa M.

Escuela de Psicología

Universidad Católica del Norte

Avenida Angamos 0610

Antofagasta,

Chile

E-mail: alurzua@ucn.cl

\section{BIBLIOGRAFÍA}

1. Gonzalez, A. Eating disorders in adolescents. Australian Family Physician. 2007; 36(8): 614-619.

2. Kreipe, R. Eating disorders in adolescents and older children. Pediatr Rev 199;20: 370 - 379.

3. Corral, S. Gonzáles, M. Pereña, J. Seisdedos, N. Manual inventario de trastornos de la conducta alimentaria EDI-2. Madrid: Tea ediciones. 2006.

4. Herrero, M. Conrado, M. Conductas y actitudes hacia la alimentación en una muestra representativa en estudiantes de secundaria. International J Clin Health Psicol 2005; 5(1): 67-83.

5. Ochoa, S. Validación y confiabilidad del inventario de trastornos de la conducta alimentaria (EDI-2) aplicado a una muestra de adolescente mexicanos. Rev Cientifica Electronica Psicol ICSa-UAEH. 2008; 6: 30-40

6. Correa, V., Zubarew, G., Silva, P., Romero, M. Prevalencia de riesgo de trastornos alimentarios en adolescentes mujeres escolares de la Región Metropolitana. Rev Chil Pediatr 2006; 77(2): 153-160.

7. Behar, R., Alvina, M., Medinellis, A.m \& Tapia, P. Trastornos de la conducta alimentaria en estudiantes de la carrera de nutrición y dietética. Rev Chil Nutr 2007; 34(4): 298 - 306.

8. Cruzat,C. Ramirez, P. Melipillan, R. Marzolo, P. Trastornos Alimentarios y Funcionamiento familiar percibido en una muestra de estudiantes secundarias 
de la comuna de Concepción, Chile. Psykhe. 2008; 17(1): 81-90.

9. Theil A.,Thomas p., Test-retest reliability of the eating disorder inventory 2. J Psychosom Res 2006; 61: 567- 569

10. García-García E, Vázquez-Velázquez V. Validez interna y utilidad diagnóstica del Eating Disorder Inventory, en mujeres mexicanas. Salud Pública Mex. 2003; 45: 206-210.

11. Garner, D.Inventario de trastornos de la conducta alimentaria EDI-2. 1983. Madrid: Tea ediciones

12. Nevonen L, Broberg AG. Validating the Eating Disorder Inventory- 2 (EDI-2) in Sweden. J Eating Weight Disord: Stud Anorexia. Bulimia Obesitas 2001;/6:/59-/67.
13. Russell, D. W. In search of underlying dimensions: the use (and abuse) of factor analysis in Personality and Social Psychology Bulletin. Personality Social Psychol Bull 2002; 28 (12): 1629-46.

14. Bentler, M. P. \& Dudgeon, P. Covariance structure analysis: Statistical practice, theory and directions. Ann Rev Psychol 1996; 47: 563-592.

15. Brown, M. W. \& Kudeck, R. Alternative ways of assesing model fit. in K. A. Bollen \& J. S. Long (Eds.), Testing structural equation models, Newbury Park, CA: Sage. pp. 136-162. 1993.

16. Scientific ADVISORY Committee of the Medical Outcomes Trust. Assessin health status and quality of life instruments: Attributtes abd review criteria. Quality Life Res 2002; 11: 193 - 205. 